\title{
Model-based Analysis of Maintenance-induced Availability of Aircraft in an Airline Network
}

\author{
Marie Bieber ${ }^{1 *}, 3$, Barbara Glock ${ }^{2}$, Alexander Plagemann ${ }^{1}$, Nikolas Popper ${ }^{3}$ \\ ${ }^{1}$ Airbus Operations $\mathrm{GmbH}$; *marietheresebieber@gmail.com \\ ${ }^{2}$ dwh GmbH simulation services, Neustiftgasse 57-59, 1070 Vienna, Austria \\ 3 Institute of Analysis and Scientific Computing, TU Wien, Wiedner Hauptstraße 8-10, 11040 Vienna, Austria;
}

SNE 30(1), 2020, 31 - 34, DOI: 10.11128/sne.30.sn.10507

Received: June 10, 2019 (Selected ASIM SST Hamburg 2018

Postconf. Publ.), Accepted: October 10, 2019

SNE - Simulation Notes Europe, ARGESIM Publisher Vienna

ISSN Print 2305-9974, Online 2306-0271, www.sne-journal.org

Abstract. Optimized aircraft maintenance concepts usually aim at increasing the aircraft availability and reducing costs. A model is presented that quantifies the effect of the increased aircraft availability when applying different maintenance concepts on an entire airline fleet. An agent-based approach is suggested: The aircraft with their maintenance tasks are dynamically assigned to the flight schedule. One major advantage of the presented methodology is that constraints for specific aircraft tails can be added easily. This is potentially of interest when testing new maintenance concept that are more focused on optimizing maintenance for single aircraft, by e.g. a stronger dependency on aircraft usage and findings.

\section{Introduction}

Currently a lot of studies are done on aircraft maintenance and its optimization to create more availability. In further consequence this leads to an economical and operational advantage for the airline operating the aircraft. Aircraft make money when they are flying. So, the baseline for airline planning is the sentence "The schedule is the king." Once the airline has flights scheduled and tickets sold, the most important thing is to operate those flights. Usually airlines plan in the following way:

1. Master Schedule design: An optimal set of flight legs is selected.

2. Fleet Assignment: The aim of the fleet assignment is to determine which type of aircraft should fly each flight leg.
3. Aircraft Scheduling: The schedule of each aircraft is determined including all maintenance tasks and the commercial constraints.

4. Tail assignment: According to the schedule and the aircraft schedules a tail number is assigned to flight legs. The master schedule is translated into an operational flight schedule.

5. Crew Assignment: To each flight leg a crew is assigned according to the working constraints of the crews.

Once the schedule is planned it is operated and continuously updated. Operational interruptions might occur and lead to delays or cancellations. This might have as a consequence changes in the operational flight schedule.

\section{Methods}

Clearly, changing the number of revenue flights means that the master schedule has to be changed. However, changing the master schedule has a big impact on airline operations and depends on a lot more factors than considered in this paper, like the airline policy. The approach used is to not change the master schedule itself but instead work with the currently used master schedule containing $\mathrm{n}$ flight legs and fix the fleet size as the smallest number of aircraft, such that all flight legs of the master schedule can be operated without any maintenance constraints. When taking into account maintenance the aircraft are able to operate $m \leq n$ flight legs of the master schedule. The number $m$ is the model output and can be used as a measurement of the effect of introducing the maintenance concept in the airline fleet. In fact, the problem that has to be dealt with is the tail assignment problem. 


\subsection{Linear Programming Approach}

In the classical approach, which is broadly used in the existing literature, the first task faced in solving the tail assignment is to identify a set of feasible flight routes that can be operated by a single aircraft. Often the maintenance constraints are already considered in this step of the tail assignment. In [1] this is described as "maintenance routing". Maintenance is often done at night due to flight restrictions. Considering this aspect [2] propose algorithms to model the aircraft routing due to these maintenance requirements. Another method to solve the maintenance routing problem is presented by [3]. As an input a fixed flight schedule with aircraft assigned to it is given, which is then optimized by reassigning the aircraft to flight routes (set of flight legs) in order to undergo different maintenance checks. A heuristic approach is provided to solve the problem of minimizing the maintenance cost and costs incurred during the reassignment of aircraft to flight segments.

The most common mathematical model used to describe the tail assignment problem is an integer linear program. This was already presented by [4]. The practise today is to combine the mathematical models with constraint programming techniques. While mathematical programming is more focused on optimization rather than on quickly obtaining a solution, constraint programming obtains a feasible solution quicker. The first approach combining the mathematical and constraints programming techniques to solve the tail assignment problem was presented by [1]. It is a very convenient approach since achieves both accuracy and is quick enough for practical use. In fact, the work has been the basis for various further studies on the tail assignment problem and is used by several airlines.

The main objective of an airline is to operate the master schedule. Operational interruptions of any kind should be avoided. The problem is examined by [5] in his doctoral thesis where two mathematical models for the robust tail assignment are proposed. They are solved by an extension of the standard solution methods presented in [1]. Another approach introduced by [6] addresses operational uncertainty by extending the oneday routes aircraft maintenance implicitly for the subsequent two days. However, here also the flight routes are given as an input, but can be modified by an iterative algorithm.

\subsection{Agent-based Modelling}

As opposed to the linear programming model, which is a top-down solution approach, an agent-based model provides a bottom-up approach. Rather than looking at the entire model system the focus lies upon each individual entity and its behaviour within the system. An agent-based system consists of one or more autonomous individuals, the agents. In [7] an 'agent' is characterized by autonomy, reactivity, pro-activeness and social ability, i.e. interaction with other agents. An agent-based model, as [8] states that 'An agent-based model is a simulation model that employs the idea of multiple agents situated and acting in a common environment as central modelling paradigm.'

The model presented in this paper is based on an agent-based approach mainly due to the adaptiveness of agent-based models. Maintenance tasks can be added to each aircraft, which allows the flexibility to define specific tasks depending on the aircraft usage or other factors. Another advantage of the agent-based approach is that instead of separating the airline planning process from the real aircraft operations, both are dynamically combined. Probabilities of delay, delay propagation and operational interruptions can bbe considered directly in the airline planning. This enables one to optimally plan airline operations in a robust and realistic way.

\section{The Agent-based Model}

The model consists of aircraft agents and the airline network represented through the master schedule that should be operated by the aircraft. The model returns the number of revenue flights operated in a specified period of time.

The main scope of the agent-based model is to show the effect of a maintenance induced increased availability of aircraft on airline operations. This is done by modelling the aircraft tails, their behaviour and movements and embedding them in the airline network. This can be seen in Figure 1, where the agents behaviour is described in a state chart. An aircraft agent can be assigned to a flight leg. After landing, if a maintenance task package is due, the aircraft undergoes maintenance. When the maintenance is finished, it is assigned a buffer time to be prepared for the next flight. If it does not undergo maintenance, the aircraft is directly assigned a buffer time when it landed. As soon as the buffer time passed, it becomes available for flights again. 


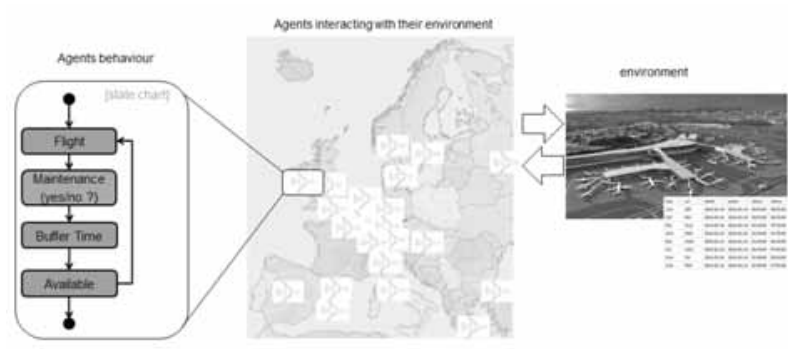

Figure 1. ABM generic architecture.

One of the characteristics of the model is that the operational flight schedule is created dynamically during a simulation run. The aircraft follow specific rules defining their behaviour. Flight legs, turn around times and maintenance tasks are assigned to the aircraft tails in the moment they occur. Therefore, not only the flight routes are created dynamically but also the ground times between flights and the maintenance ground times.

\section{Results}

Based on in-service data and real airline data the model was tested for various maintenance scenarios. One of those scenarios aimed at analysing the effect of check overruns. Scheduled maintenance includes regulated maintenance tasks, that have to be performed regularly. The most common maintenance packaging practise is to perform checks in regular intervals, such as daily or weekly checks. A typical task packaging setup is illustrated in Table 1.

\begin{tabular}{lll}
\hline maintenance type & frequency (days) & duration (hours) \\
\hline daily & 1.5 & 2 \\
\hline weekly & 8 & 3 \\
\hline A & 190 & 8 \\
\hline $1 C$ & 730 & 93 \\
\hline $2 C$ & 1460 & 140 \\
\hline $6 Y$ & 2190 & 350 \\
\hline $12 Y$ & 4380 & 730 \\
\hline
\end{tabular}

Table 1. Typical scheduled block type maintenance packages.

The term check overrun refers to the phenomena that performing a maintenance task package took for an unexpected reason longer than it was scheduled. But does reducing these overruns have a considerable impact on airline operations?
The scenario of a $1 \mathrm{C}$ check overrun is simulated for an example airline with a calibrated fleet size of 31 aircraft. Instead of the usual duration of 93 hours one assumes the check to take 98 hours (which equals an overrun of around 0.2 days). The overall results in terms of the number of revenue flights and the average ground times for the simulated period of time are given in Table 2 .

\begin{tabular}{llc}
\hline & no overrun & overrun \\
\hline $\begin{array}{l}\text { Number of revenue } \\
\text { flights }\end{array}$ & 6325 & 6325 \\
\hline $\begin{array}{l}\text { Average ground } \\
\text { time (seconds) }\end{array}$ & 12100.29 & 12095.13 \\
\hline
\end{tabular}

Table 2. Results for the Scenario 1C Check overrun.

In Figure 2 the number of revenue flights for every aircraft tail are listed where the tails that had to undergo a 1C checks are highlighted. Similarly, Figure 3 displays the average ground times for every aircraft tail.

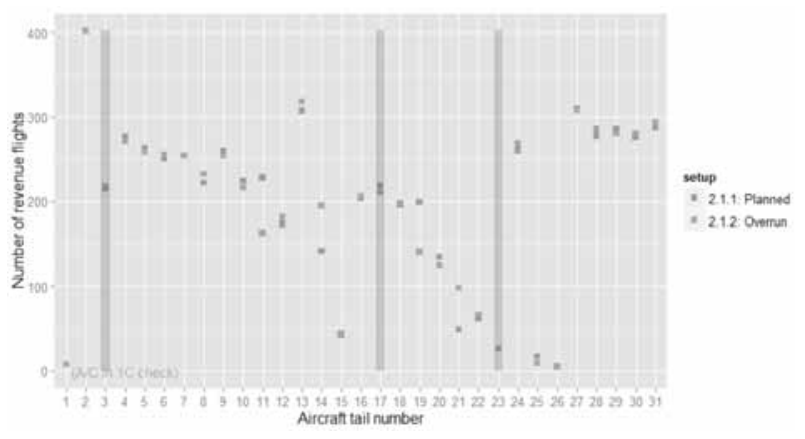

Figure 2. Number of revenue 2ights per aircraft tail for the $1 \mathrm{C}$ check overrun.

The simulation reveals that increasing the duration for a $1 \mathrm{C}$ check overrun does not have a significant impact, neither on the total number of revenue flights operated during the time frame nor on the average ground times. For the aircraft tails that had to undergo the $1 \mathrm{C}$ check the results are more in alignment to what is expected: For two aircraft tails the number of revenue flights decreased in the overrun setup and the average ground time increased.

A reasonable explanation for this is that the model dynamics causes an aircraft that is delayed unexpectedly to be replaced by another aircraft dynamically. As a consequence, delays are compensated directly and with a simplicity that is not feasible in reality. The fleet size of the airline is big enough to compensate a downtime of a single aircraft tail efficiently. 


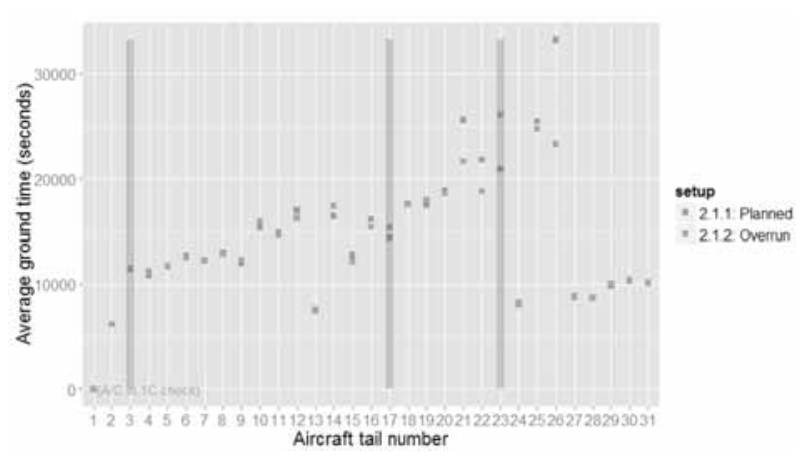

Figure 3. Average ground times per aircraft tail for the $1 C$ check overrun.

\section{Challenges and Future Developments}

During simulations it was observed that some unexpected results are due to a poor fleet size calibration. As a next step, it would be suggested to work on that matter.

One major limitation of the model was observed to be how it handles an unexpected delay, in this case for maintenance tasks. Rather than reflecting the effect of the very complicated process of adopting to unexpected changes, as it would happen in real airline operations, the model assigns a different aircraft tail to the flight that should be operated and therefore dynamically solves the interruption caused in airline operations. To see the effects of e.g. an overrun as it happens in reality better, a possibility would be to first run the model and than use the thereby created tail assignment as a constraint for the second run, where the aircraft operations are simulated.

\section{Conclusion}

The model performs quite well when tuning the input parameters to be very close to what is used for real flight operations. This is also true for the maintenance setup.
When changing the maintenance scenario significantly, the model adopts to the new setup. Then, the results for the number of operated revenue flights and average ground times are much closer to the classical maintenance scenario than what is observed for real flight operations.

All in all, the approach has the potential to be developed further for performing tail assignments that are adoptable to a wide range of maintenance scenarios and is adaptive to changes. Through using an agent-based approach a new methodology was devised that provides an alternative to the classical linear programming approach. In the agent-based approach constraints and attributes can be altered for single aircraft tails. One promising application for the model would be to analyze the effect of maintenance concepts that are more dependent on the usage in flight hours or flight cycles or individual findings or failures for single aircraft tails. The paper provides the framework for further investigations into this field.

\section{References}

[1] Grönkvist M. The tail assignment problem. Chalmers tekniska högskola. 2005.

[2] Gopalan R, Talluri KT. The Aircraft Maintenance Routing Problem. Operations Research. 1998; 46:260-271.

[3] Sriram C, Haghani A. An optimization model for aircraft maintenance scheduling and re-assignment. Transportation Research Part A: Policy and Practice. 2003;37(1):29-48.

[4] Levin A. Scheduling and fleet routing models for transportation systems. Transportation Science. 1971; 5(3):232-255.

[5] Dovica I. Robust tail assignment. 2014;

[6] Maher SJ, Desaulniers G, Soumis F. The tail assignment problem with look-ahead maintenance constraints. 2015;

[7] Wooldridge M. Agent-based software engineering, vol. 144. 1997.

[8] Siegfried R. Modeling and simulation of complex systems: A framework for efficient agent-based modeling and simulation. Springer. 2014. 